\title{
Article
}

\section{Electrohydrodynamic Instabilities in Free Emulsion Films}

\author{
Farshid Mostowfi ${ }^{1}{ }^{\oplus}$, Plamen Tchoukov ${ }^{2, *}$, Nikolay Panchev ${ }^{2}$, Tadeusz Dabros ${ }^{3}$ and Jan Czarnecki ${ }^{4}$ \\ 1 Schlumberger Doll-Research, Cambridge, MA 02139, USA; FMostowfi@slb.com \\ 2 Institute of Physical Chemistry, Bulgarian Academy of Sciences, 1113 Sofia, Bulgaria; npanchev@ipc.bas.bg \\ 3 Natural Resources Canada, 1 Oil Patch Drive, Devon, AB T9G 1A8, Canada \\ 4 Department of Chemical and Materials Engineering, University of Alberta, Edmonton, AB T6G 2G6, Canada; \\ jc7@ualberta.ca \\ * Correspondence: tchoukov@ualberta.ca or tchoukov@ipc.bas.bg
}

Citation: Mostowfi, F.; Tchoukov, P.; Panchev, N.; Dabros, T.; Czarnecki, J. Electrohydrodynamic Instabilities in Free Emulsion Films. Colloids Interfaces 2021, 5, 36. https:// doi.org/10.3390/colloids5030036

Academic Editor: Georgi G. Gochev

Received: 21 April 2021

Accepted: 28 June 2021

Published: 1 July 2021

Publisher's Note: MDPI stays neutral with regard to jurisdictional claims in published maps and institutional affiliations.

Copyright: (c) 2021 by the authors. Licensee MDPI, Basel, Switzerland. This article is an open access article distributed under the terms and conditions of the Creative Commons Attribution (CC BY) license (https:/ / creativecommons.org/licenses/by/ $4.0 /)$.

\begin{abstract}
Electrohydrodynamic instabilities were induced in thin water-in-oil emulsion films by application of external DC electric field. The dominant wavelengths of instabilities were measured for constant electric fields of various strengths. The dominant wavelengths agreed reasonably well with theoretical predictions based on a linear stability model. The linear stability model used in this study took into account experimentally measured repulsive disjoining pressure and calculated Maxwell stress. The observation of such instabilities can help to understand the rupture mechanism of emulsion films under the influence of electric field.
\end{abstract}

Keywords: electrohydrodynamic instabilities; water-in-oil emulsion films; electrocoalescence; linear stability model; thin film breakdown

\section{Introduction}

Electric-field-induced breakdown of emulsion films has broad applications in industrial and biological systems. In the petroleum industry, for instance, electrocoalescence is widely used to demulsify water-in-oil emulsions [1-4]. Formation of pores in bilayer lipid membranes (BLM) using electric fields (electroporation) has also been used to transfer molecules into the cell body $[5,6]$. While the electric-field-induced breakdown of bilayer lipid membranes is well studied, the breakdown mechanism for common emulsion films encountered in industry is still not well understood. Electroporation in emulsion films is unlikely because they are rather thick. Emulsion films consist of two adsorbed layers with a considerable solvent core. The thickness of these films is on the order of tens of nanometers [7-9]. Bilayers, on the other hand, are much thinner, typically a few nanometers, and virtually without a solvent core [10]. Furthermore, pore nucleation in bilayers is experimentally detectable by a sudden increase in conductance of the film at the onset of pore formation but has not been reported for typical emulsion films [11,12]. Dielectric breakdown, which manifests as the growth of conduction paths through a nonconductive medium beyond a critical electric field strength, is another possible mechanism. However, the dielectric strengths of typical organic solvents are an order of magnitude greater than those required for the breakdown of thin films [13].

Electric-field-induced instabilities have been observed in wetting films, where a thin liquid film is squeezed between a solid substrate and air. Electric fields have been used as a means to transfer patterns onto polymer films [14-16]. However, experimental evidence for the existence of such instabilities in free emulsion films is scarce [17]. Herein, we report evidence of electric-field-induced instabilities in free water-in-oil emulsion films. The dominant wavelengths of these instabilities were measured as a function of applied DC (direct current) electric fields strengths. Experimentally measured disjoining pressure and calculated Maxwell stress are used in a linear stability model to interpret the experimental results for dominant wavelengths. 


\section{Materials and Methods}

\subsection{Materials}

The oil phase was a solution of Tegopren 7008 (Degussa, Essen, Germany) in ndecane (Fisher Scientific, Pittsburgh, PA, USA). Tegopren 7008 is a high-molecular-weight ( $>10,000 \mathrm{Da}$ ) block copolymer with strong emulsifying properties for water-in-oil type emulsions [18]. The oil phase was prepared at two different Tegopren 7008 concentrations: $0.058 \mathrm{wt} . \%$ ("film A") and $0.52 \mathrm{wt} . \%$ ("film B"). The aqueous phase was $1 \mathrm{wt.} \%$ solution of sodium chloride in Milli-Q water, $18.2 \mathrm{M} \Omega \cdot \mathrm{cm}$.

The porous glass plate was treated hydrophobic by soaking it for $24 \mathrm{~h}$ in a solution of $20 \mathrm{wt} . \%$ dichlorodimethylsilane (purity $>99.5 \%$, Fluka) in cyclohexene (reagent grade, Fisher Scientific, Pittsburgh, PA, USA).

\subsection{Experimental Setup}

A schematic diagram of the experimental setup is shown in Figure 1. A single thin emulsion film is formed in the modified Scheludko-Exerowa cell that allows for application of DC or AC (alternating current) polarizations to single water-in-oil emulsion films [19]. The cell consisted of a hydrophobic porous glass plate, in which a small hole has been drilled to hold the film. The porous plate, soaked with oil, separated two aqueous phases (electrically insulated). Both aqueous phases were de-ionized water with addition of sodium chloride ( $1 \mathrm{wt} . \%)$ to increase the conductivity of the solutions. Platinum wire electrodes were placed in the aqueous phases. The electrodes were connected to a Solartron 1287 potentiostat (Solartron, Farnborough, UK) and used to apply DC electric field. The films were observed and recorded in reflected light with Zeiss Axio Observer microscope and Leica DFC500 digital camera. The disjoining pressure isotherms were measured by applying external pressure on the film and measuring the film thickness. The external pressure was controlled via Hamilton Syringe Drive PSD/2 and pressure transducer from Omega. The film thickness was measured following conventional interferometry techniques used in thin-film applications [20,21]. Hg-arc lamp, a green interference filter $546 \mathrm{~nm}$, and a low-noise Hamamatsu Si photodiode S8745-01 were used for registration of photo signal.

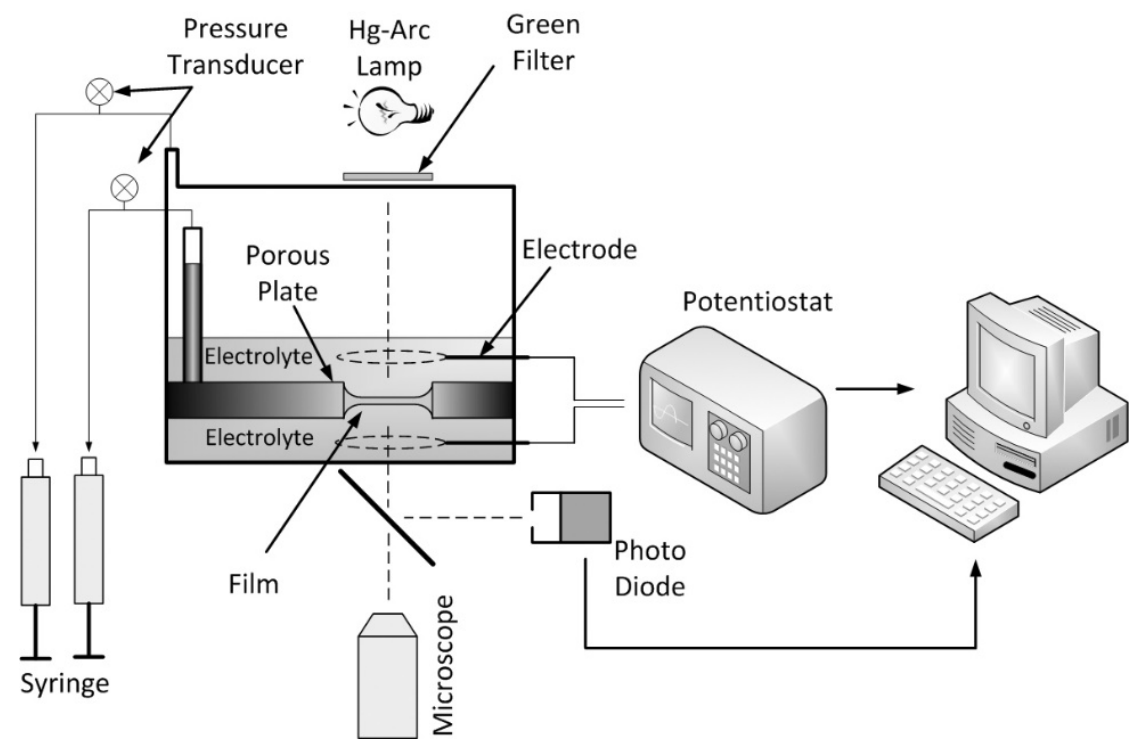

Figure 1. Schematic diagram of the experimental setup.

\section{Results and Discussion}

Both Tegopren 7008 concentrations, "film A" (0.058 wt.\%) and "film B" (0.52 wt.\%) formed stable films with long lifetimes and good stability against applied electric fields. The thin films were obtained by slowly withdrawing oil from the porous plate through a 
capillary until a plane parallel film was formed. The films were allowed to drain for at least $30 \mathrm{~min}$ to achieve the final equilibrium thicknesses before any electric field was applied. The equilibrium thicknesses of films A and B were 57 and $72 \mathrm{~nm}$, respectively.

Figure $2 \mathrm{a}$ is a micrograph of a film at rest. Relatively uniform light distribution across the plane of the film indicates uniform film thickness. Once the films reached their equilibrium thicknesses, a step potential was applied across the film through platinum electrodes using the Solartron 1287 potentiostat. Upon applying the electric field, instabilities appeared in a form of small white dots or ripples as shown in Figure $2 b, c$. In these pictures, the white dots represent thicker film areas. To study the behavior of the films, step electric potentials varying from 0 to $900 \mathrm{mV}$ with $100 \mathrm{mV}$ increments were applied. Each measurement started with a uniform and well-equilibrated film, as shown in Figure 2a. No instabilities were observed below $200 \mathrm{mV}$, while the films ruptured at potentials above $900 \mathrm{mV}$. Once the electric field was removed, the instabilities disappeared, and the films reestablished their uniform thickness at equilibrium state. When a constant electric field was maintained, however, the white spots slowly merged, mostly through a mode similar to the coarsening process observed in wetting films [22]. A few coalescence events were also observed. The images shown in Figure $2 b, c$ correspond to the first frames captured right after applying the potential.

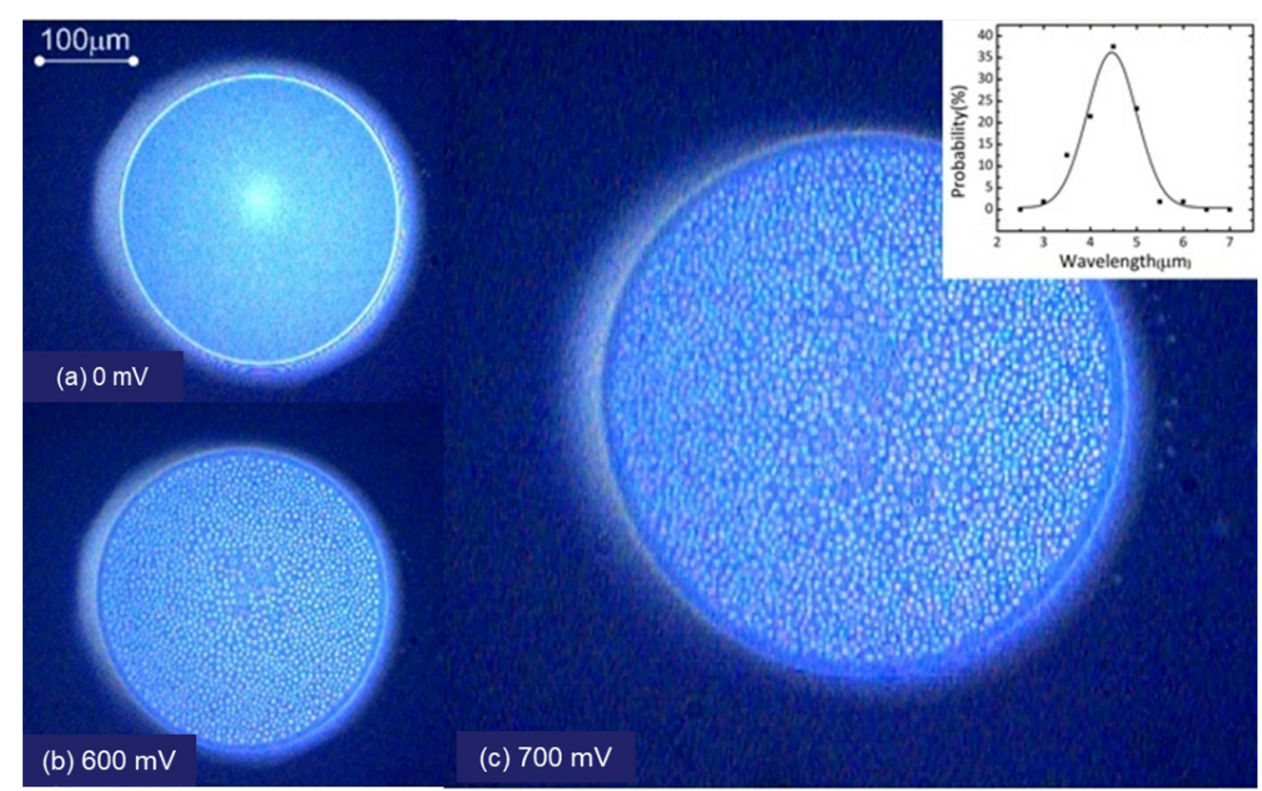

Figure 2. (a) Film at equilibrium, $U=0 \mathrm{mV}$; (b) instabilities that appeared on the plane of the film when an electric potential was applied, $U=600 \mathrm{mV}$; and (c) instabilities at $U=700 \mathrm{mV}$, the inset shows the nearest-neighbor distribution of the white spots.

The comparison of images obtained at different conditions showed a clear correlation between the applied potential and the distribution of the spots: the stronger the electric field, the greater the number density of the spots. To characterize the instabilities, we measured the nearest neighbor-to-neighbor distance distribution of the spots. The inset in Figure $2 \mathrm{c}$ shows the distribution of the measured distances. The maximum of the normal distribution of the distances was considered to be the dominant or the fastest-growing wavelength for the instabilities.

The dominant wavelength, $\lambda_{\max }$, corresponding to the applied potentials is plotted in Figure 3. It is assumed that the applied potential across the electrodes translates into equivalent electric potential across the film. This assumption is justified given the amount of electrolyte in the aqueous phase, thus its high electric conductivity, compared to very low conductivity of the oily film liquid. Electrode polarization is neglected due to the low current (current density) flowing through the system. The figure shows a reduction 
in the wavelength of the fastest-growing instabilities when the potential is increased. Furthermore, the dominant wavelengths for both systems (i.e., for film A and film B) are similar; in particular, they are indistinguishable at potentials greater than $400 \mathrm{mV}$.

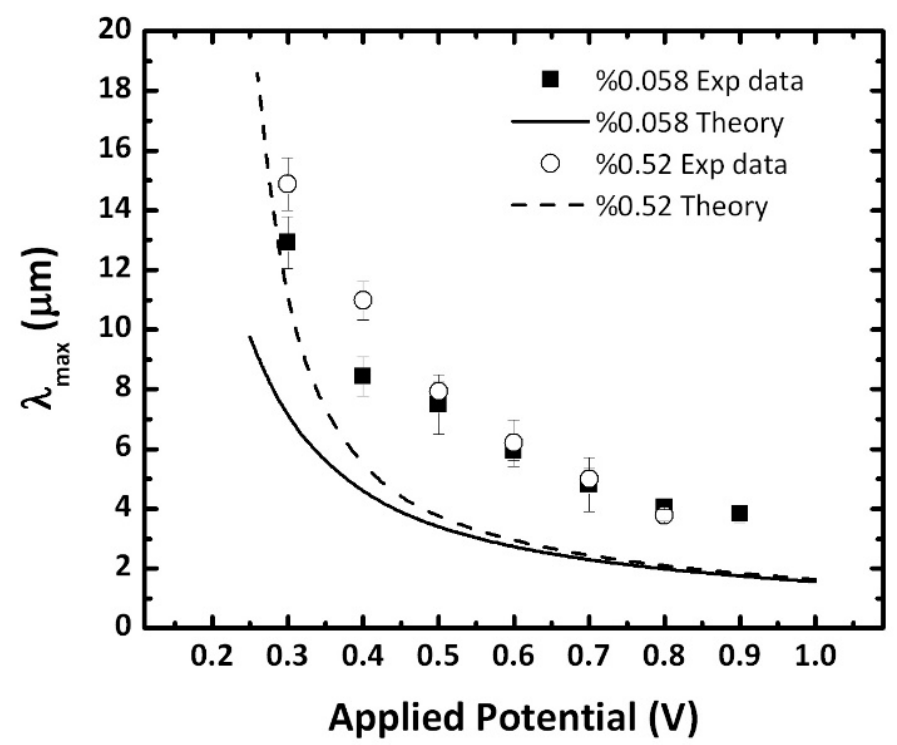

Figure 3. The dominant wavelength of the emulsion film for various applied potentials. The graph compares the calculated and measured dominant wavelengths for various field strengths.

We explain this observation by using linear stability theory [23-26], taking into account the surface forces acting in the film. Before using the linear stability theory, one needs to emphasize its underlying assumption. In this theory, the Navier-Stokes equations are linearized assuming the amplitude of the instabilities is much smaller than the thickness of the film. Despite this approximation, the linear model provides a simple and straightforward solution that explains the underlying mechanism of electric-field-induced rupture [23-25].

Now, let us analyze the pressure difference, $\Delta p$, between the perturbed state and the initial state before the application of electric field. If we follow Mileva and Radoev [26], $\Delta p$ can be presented as:

$$
-\Delta p=p_{\gamma}(\delta)+P_{S T}(h+2 \delta)-P_{S T}(h)
$$

where $p_{\gamma}, \delta, h$ are the local capillary pressure, the displacement of film surface from initial position due to instability, and the initial film thickness, respectively. $P_{S T}(h+2 \delta)$ and $P_{S T}(h)$ are stabilizing pressures acting on the film. In our case, we have two major pressure contributions: disjoining pressure $\Pi(h)$ which includes all surface forces acting in the film (e.g., Van der Waals, electrostatic, and steric forces) and Maxwell stress due to the applied electric field.

$$
P_{s t}(h)=\Pi(h)-\frac{1}{2} \varepsilon\left(\frac{U}{h}\right)^{2}
$$

In Equation (2), $\varepsilon$ and $U$ are the permittivity of oil phase and applied potential, respectively. $\Pi(h)$ by definition is positive when stabilizing the film and the Maxwell stress is acting in the opposite direction. By assuming an immobile film interfaces due to the adsorption of the surfactant molecules and performing perturbation analysis followed by Fourier transformation $[25,26]$, one can obtain the following expression for the wavenumber of the fastest-growth instability mode, $q_{\max }$ :

$$
q_{\max }^{2}=\frac{\left(\frac{d P_{s t}}{d h}\right)_{h_{0}}}{\gamma}=\frac{1}{\gamma}\left\{\left(\frac{d \Pi}{d h}\right)_{h_{0}}+\frac{\varepsilon U^{2}}{h_{0}^{3}}\right\}
$$


where $\gamma, h_{0}$ are the interfacial tension and equilibrium film thickness. As shown by Herminghaus [23], for wetting films with no surfactant, the contribution of Van der Waals force to the dominant wavelength is negligible compared to that of an electric field. In an emulsion film with adsorbed surfactant layer, however, the contribution of repulsive interactions should be taken into account. To quantify the contribution of stabilizing surface forces, disjoining pressures of the films were measured, and the results are shown in Figure 4. In the measurement of the disjoining pressures, no electric force was applied. Therefore, the disjoining pressure consists of all interactions including Van der Waals, electrostatic (due to surface charges), and steric forces. We used the slope of the linear fit of the experimental data points in proximity of $h_{0}$ as an estimate of the derivative of the disjoining pressure at the equilibrium film thickness, as shown in the Figure 4.

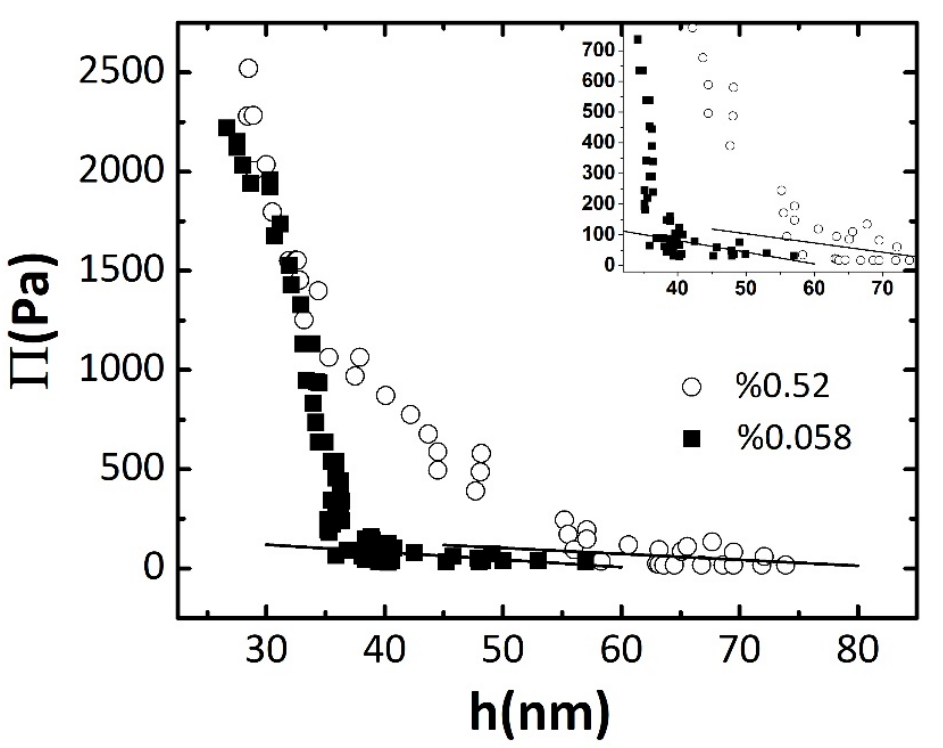

Figure 4. Disjoining pressure vs. film thickness for films A and B. A straight line is fitted to the experimental data to obtain the derivative of the disjoining pressure.

Now, one may calculate the fastest-growing wavelengths of the instabilities $\left(\lambda_{\max }=2 \pi\right.$ ) $q_{\max }$ ) using Equation (3). These are shown by the solid and dashed lines in Figure 3 for films A and B, respectively. Considering limitations of linear stability theory, a reasonable agreement between theory and the experimental data is achieved. The model follows the trend of the experimental data. Furthermore, the model shows that almost identical dominant wavelengths are expected for potentials greater than $400 \mathrm{mV}$. The relatively high interfacial tension in film A is counterbalanced by the greater slope of disjoining pressure vs. film thickness and lower film thickness, yielding a dominant wavelength similar to that for film B. Table 1 shows the experimental data for film thickness, interfacial tension, and derivative of the disjoining pressure used in the model.

Table 1. Experimental data for film thickness, interfacial tension, and the derivative of disjoining pressure that were used in the model.

\begin{tabular}{cccc}
\hline Conc. (wt.\%) & $\begin{array}{c}\text { Equilibrium } \\
\text { Thickness } \mathbf{( n m})\end{array}$ & $\begin{array}{c}\text { Equilibrium Interfacial } \\
\text { Tension }\left(\mathbf{m J} / \mathbf{m}^{2}\right)\end{array}$ & $\frac{\partial \Pi}{\partial h}(\mathbf{P a} / \mathbf{n m})$ \\
\hline 0.058 & $57 \pm 1$ & $5.8 \pm 0.5$ & -3.8 \\
0.52 & $72 \pm 1$ & $3.2 \pm 0.5$ & -3.0 \\
\hline
\end{tabular}


The linear stability model allows to calculate the critical wavenumber and wavelength of the instability:

$$
q_{c r}=\frac{2 \pi}{\lambda_{c r}}=\left[\frac{2}{\gamma}\left\{\left(\frac{d \Pi}{d h}\right)_{h_{0}}+\frac{\varepsilon U^{2}}{h_{0}^{3}}\right\}\right]^{\frac{1}{2}}
$$

Since $(d \Pi / d h)_{h 0}<0$, the value of $q_{c r}$ has a real value if $\varepsilon U^{2} / h_{0}^{3}>\left|(d \Pi / d h)_{h_{0}}\right|$. When $q_{c r}$ is real, for a given electric field, there exist wavelengths that destabilize the film (those with $\left.\lambda>\lambda_{c r}\right)$. However, for electric potentials that make $\varepsilon U^{2} / h_{0}^{3}<\left|(d \Pi / d h)_{h_{0}}\right|, q_{c r}$ has no real roots. This condition is satisfied for electric potentials below 0.19 and $0.25 \mathrm{~V}$ for films $\mathrm{A}$ and $\mathrm{B}$, respectively. In other words, the film is stable for all wavelengths at potentials below these values due to the stabilizing repulsive disjoining pressure. This corroborates our observations-no instabilities were detected below $0.2 \mathrm{~V}$.

\section{Conclusions}

Upon application of an electric field across water-oil-water emulsion films, instability patterns were observed as small white dots in the film's microscopic images. The characteristic lengths of these instabilities were measured for constant electric fields of various strengths. The experimentally observed dominant and critical wavelengths agreed reasonably well with theoretical predictions based on the linear stability model. The linear stability model used in this study took into account experimentally measured disjoining pressure and calculated Maxwell stress. The reported results demonstrated that the formation of electrohydrodynamic instabilities is a possible mechanism for breakup of thick emulsion films. DC polarization studies of single thin emulsion films are a promising approach for understanding of electro-induced coalescence of $\mathrm{W} / \mathrm{O}$ emulsions.

Author Contributions: Conceptualization, J.C., F.M. and N.P.; methodology, P.T., F.M. and J.C.; formal analysis, F.M. and P.T.; investigation, P.T.; writing-original draft preparation, F.M.; writingreview and editing, P.T., J.C., N.P. and T.D.; visualization, F.M.; supervision, T.D., J.C.; F.M., P.T., N.P. and J.C. have read and agreed to the published version of the manuscript. Sadly, our colleague and friend T.D. passed away before this submission and was not able to review the published version. However, he reviewed and approved a previous version of the manuscript containing all results and conclusions included in the final manuscript.

Funding: This research received no external funding.

Data Availability Statement: Not applicable.

Acknowledgments: Authors would like to acknowledge Radomir Slavchov, Queen Mary University of London; Richard Craster, Imperial College London; and Khristo Khristov, Institute of Physical Chemistry, Bulgarian Academy of Sciences, for helpful discussions.

Conflicts of Interest: The authors declare no conflict of interest.

\section{References}

1. Taylor, S.E. Investigations into the electrical and coalescence behaviour of water-in-crude oil emulsions in high voltage gradients. Colloids Surf. 1988, 29, 29-51. [CrossRef]

2. Eow, J.S.; Ghadiri, M.; Sharif, A.O.; Williams, T.J. Electrostatic enhancement of coalescence of water droplets in oil: A review of the current understanding. Chem. Eng. J. 2001, 84, 173-192. [CrossRef]

3. Narve, A.; Kallevik, H.; Sjöblom, J. Water-in-crude oil emulsion stability studied by critical electric field measurements. Correlation to physico-chemical parameters and near-infrared spectroscopy. J. Pet. Sci. Eng. 2002, 36, 1-17.

4. Sameer, M.; Simon, S.; Sjöblom, J.; Xu, Z. Demulsifier assisted film thinning and coalescence in crude oil emulsions under DC electric fields. Chem. Eng. Res. Des. 2018, 134, 117-129.

5. Abidor, I.G.; Arakelyan, V.B.; Chernomordik, L.V.; Chizmadzhev, Y.A.; Pastushenko, V.F.; Tarasevich, M.P. Electric breakdown of bilayer lipid membranes: I. The main experimental facts and their qualitative discussion. J. Electroanal. Chem. Interf. Electrochem. 1979, 104, 37-52. [CrossRef]

6. Weaver, J.C.; Chizmadzhev, Y.A. Theory of electroporation: A review. Bioelectrochem. Bioenerg. 1996, 41, 135-160. [CrossRef]

7. Czarnecki, J.; Khristov, K.; Masliyah, J.; Panchev, N.; Taylor, S.D.; Tchoukov, P. Application of Scheludko-Exerowa thin liquid film technique to studies of petroleum W/O emulsions. Colloids Surf. A 2017, 519, 2-10. [CrossRef] 
8. Khristov, K.; Taylor, S.D.; Czarnecki, J.; Masliyah, J. Thin liquid film technique-Application to water-oil-water bitumen emulsion films. Colloids Surf. A 2000, 174, 183-196. [CrossRef]

9. Tchoukov, P.; Czarnecki, J.; Dabros, T. Study of water-in-oil thin liquid films: Implications for the stability of petroleum emulsions. Colloids Surf. 2010, 372, 15-21. [CrossRef]

10. Huang, C.; Thompson, T.E. Properties of lipid bilayer membranes separating two aqueous phases: Determination of membrane thickness. J. Mol. Biol. 1965, 13, 183-193. [CrossRef]

11. Mostowfi, F.; Khristov, K.; Czarnecki, J.; Masliyah, J.; Bhattacharjee, S. Electric field mediated breakdown of thin liquid films separating microscopic emulsion droplets. Appl. Phys. Lett. 2007, 90, 184102. [CrossRef]

12. Mostowfi, F.; Czarnecki, J.; Masliyah, J.; Bhattacharjee, S. A microfluidic electrochemical detection technique for assessing stability of thin films and emulsions. J. Colloid. Intref. Sci. 2008, 317, 593-603. [CrossRef]

13. Priest, C.; Herminghaus, S.; Seemann, R. Controlled electrocoalescence in microfluidics: Targeting a single lamella. Appl. Phys. Lett. 2006, 89, 134101. [CrossRef]

14. Schäffer, E.; Thurn-Albrecht, T.; Russell, T.P.; Steiner, U. Electrically induced structure formation and pattern transfer. Nature 2000, 403, 874. [CrossRef] [PubMed]

15. Schäffer, E.; Thurn-Albrecht, T.; Russell, T.P.; Steiner, U. Electrohydrodynamic instabilities in polymer films. Eur. Phys. Lett. 2001, 53, 518. [CrossRef]

16. Deshpande, P.; Pease, L.F.; Chen, L.; Chou, S.Y.; Russel, W.B. Cylindrically symmetric electrohydrodynamic patterning. Phys. Rev. E 2004, 70, 041601. [CrossRef]

17. Oron, A.; Davis, S.H.; Bankoff, S.G. Long-scale evolution of thin liquid films. Rev. Mod. Phys. 1997, 69, 931. [CrossRef]

18. O'Sullivan, M.F. Microcapsules with Liquid Cores and Solid Shells for Pressure Release Applications. Ph.D. Thesis, University of Bristol, Bristol, UK, 2007.

19. Panchev, N.; Khristov, K.; Czarnecki, J.; Exerowa, D.; Bhattacharjee, S.; Masliyah, J. A new method for water-in-oil emulsion film studies. Colloids Surf. A 2008, 315, 74. [CrossRef]

20. Scheludko, A.; Platikanov, D. Utersuchung dünner Flüssinger Schichten auf Quecksiber. Kolloid Z. 1961, 175, 150-158.

21. Sheludko, A. Thins Liquid Films. Adv. Colloid Interface Sci. 1967, 1, 335. [CrossRef]

22. Mitlin, V.S.; Petviashvili, N.V. Nonlinear dynamics of dewetting: Kinetically stable structures. Phys. Lett. A 1994, $192,323$. [CrossRef]

23. Herminghaus, S. Dynamical Instability of Thin Liquid Films Between Conducting Media. Phys. Rev. Lett. 1999, 83, 2359. [CrossRef]

24. Bandyopadhyay, D.; Sharma, A. Electric field induced instabilities in thin confined bilayers. J. Colloid Interface Sci. 2007, 311, 595. [CrossRef] [PubMed]

25. Ruckenstein, E.; Jain, R.K. Spontaneous rupture of thin liquid films. J. Chem. Soc. Faraday Trans. 1974, 70, 132. [CrossRef]

26. Mileva, E.; Radoev, B. Hydrodynamic interactions and stability of emulsion films. In Emulsions: Structure Stability and Interactions; Elsevier: New York, NY, USA, 2004; pp. 215-258. 